Communications in Physics, Vol. 19, No. 4 (2009), pp. 222-228

\title{
ENTANGLEMENT OF A SCATTERED SINGLE PHOTON AND AN EXCITON
}

\author{
NGUYEN DUC GIANG, TRAN THI THANH VAN, NGO VAN THANH, \\ AND NGUYEN AI VIET \\ Institute of Physics, VAST
}

\begin{abstract}
A single photon which is initially uncorrelated with an exciton will evolve to be entangled with the exciton on their continuous kinetic variables in the process of resonant scattering. We find the relations between the entanglement and their physical control parameters, which indicates that high entanglement can be reached by changing specific parameters of exciton.
\end{abstract}

\section{INTRODUCTION}

Entanglement with continuous variable attracts many attentions for its fundamental importance in quantum nonlocality [1] and quantum information science and technology [2]. As a physical realization, the continuous momentum entanglement between atom and photon has been extensively studied in the recent years [3-6]. In the process of resonant scattering [5], the momentum conservation will induce the atom-photon entanglement with the degree is proportional to the momentum variance. Based on the similarities in excitons and atoms, in this paper, we propose a novel scheme to control the entanglement between a single exciton and a photon. Due to the specific behaviors of the excitons, this new mechanism will exhibit interesting features of entanglement.

To describe the degree of entanglement, we calculate first the ratio $R$ between the conditional and unconditional variance of momentum to evaluate the two particles correlations in the probability amplitude of their wave function, which is experimentally accessible and can be seen as the "amplitude entanglement" in the momentum space [5]. Then we use the standard Schmidt decomposition $[8,9,12]$ and treat the Schmidt number $K[9]$ as a criterion for the full entanglement contained both in amplitude and phase. As in the previous system [5], for both criteria $R$ and $K$, we revealed their similar dependences on the physical control parameters of the system. However, due to the difference of the excitons, we study the parameters in completed different scales to evaluate the degree of entanglement.

\section{THEORETICAL MODEL}

As shown in Fig. 1(a), the two-level exciton in semiconductors has transition frequency $\omega_{e}$ and total mass $m$, the ground state and excited state are denoted by $|1\rangle$ and 


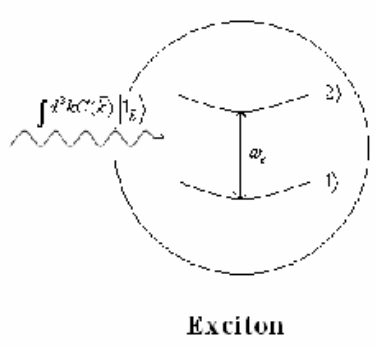

(a)

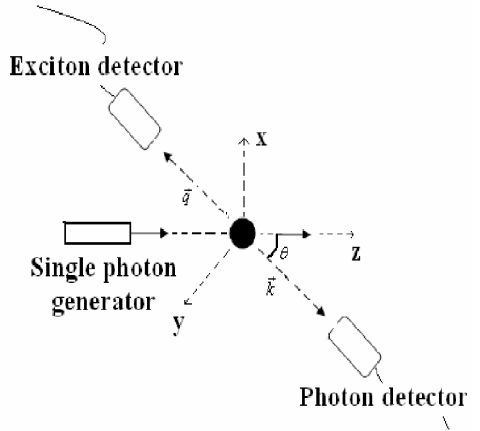

(b)

Fig. 1. (a) Single photon interacts resonantly with free two-level exciton. (b) The incident photon is scattered by the exciton; angle $\theta$ is fixed to determine the direction of the detection.

$|2\rangle$, respectively. The incident single photon from some generator is resonant with the exciton and exhibits a superposed state of different Fock states due to its linewidth. We fix the photon detector and exciton detector in opposite directions and make them both in the $x-z$ plane for simplicity (see in Fig. 1(b)); the angle $\theta$ can be controlled to observe the scattering in needed directions. The Hamiltonian of this system under the rotating wave approximation (RWA) could be written as:

$$
\hat{H}=\frac{(\hbar \hat{p})^{2}}{2 m}+\sum_{\vec{k}} \hbar \omega_{\vec{k}} \hat{a}_{\vec{k}}^{+} \hat{a}_{\vec{k}}+\hbar \omega_{e} \hat{\sigma}_{22}+\hbar \sum_{\vec{k}}\left[g(\vec{k}) \omega_{e} \hat{\sigma}_{12} \hat{a}_{\vec{k}}^{+} e^{-i \vec{k} \cdot \vec{r}}+H . c .\right]
$$

where $\hbar \hat{p}$ and $\vec{r}$ denote the center of mass momentum and position operators of the exciton, $\hat{\sigma}_{i j}$ denotes the excitonic transition operator $|i\rangle\langle j|$ where $i, j=1,2 ; \hat{a}_{\vec{k}}$ and $\hat{a}_{\vec{k}}^{+}$are the annihilation and creation operators for the light mode with the photonic wave vector $\vec{k}$ and frequency $\omega_{\vec{k}}=c k$, respectively. Note that the summation is performed over all coupled modes in the continuous Hilbert space. We also suppress the polarization index in the summation as well as in the photon state, since we can always choose a particular polarization to detect the photon. $g(\vec{k})$ is the dipole coupling coefficient.

As there is only one photon in the interaction, the basis of the Hilbert space can be denoted as $\left|\vec{q}, 1_{\vec{k}, i}\right\rangle(\mathrm{i}=1,2)$, where the arguments in the kets denote, respectively, the wave vector of the exciton and of the photon, and the excitonic internal state. At time $t$ the state vector can therefore be expanded as

$$
|\psi\rangle=\sum_{\vec{q}, \vec{k}} C_{1}(\vec{q}, \vec{k}, t)\left|\vec{q}, 1_{\vec{k}}, 1\right\rangle+\sum_{\vec{q}} C_{2}(\vec{q}, t)|\vec{q}, 0,2\rangle
$$


Substituting Eqs.(1) and (2) into the Schrödinger equation yields

$$
\begin{aligned}
i \dot{A}(\vec{q}, \vec{k}, t) & =g(\vec{k}) B(\vec{q}+\vec{k}, t) e^{i\left[c k-\omega_{e}-(\hbar / 2 m)(2 \vec{q}+\vec{k}) \cdot \vec{k}\right] t}, \\
i \dot{B}(\vec{q}, t) & =\sum_{\vec{k}} g^{*}(\vec{k}) A(\vec{q}-\vec{k}, \vec{k}, t) e^{i\left[\omega_{e}-c k+(\hbar / 2 m)(2 \vec{q}-\vec{k}) \cdot \vec{k}\right] t},
\end{aligned}
$$

in which $A, B$ are the slow varying parts of $C_{1}$ and $C_{2}$, i.e.,

$$
\begin{aligned}
A(\vec{q}, \vec{k}, t) & =C_{1}(\vec{q}, \vec{k}, t) e^{i\left(\hbar q^{2} / 2 m+c k\right) t}, \\
B(\vec{q}, t) & =C_{2}(\vec{q}, t) e^{i\left(\hbar q^{2} / 2 m+\omega_{e}\right) t} .
\end{aligned}
$$

The exciton is initially prepared in state $|1\rangle$ with the momentum wave-function is defined by $G_{j}\left(q_{j}\right)=e^{-\left(q_{j} / \delta q_{j}\right)^{2}}$, with $j=x, y, z$, in which $\delta q$ denotes its momentum variance. If the distance between the incident photon and exciton is $L$, one can choose the photonic wave function as $P_{j}=e^{i \phi\left(k_{j}, L\right)} /\left(k_{j} / \delta k_{j}+i\right)$ with $j=x, y, z$, which is a good approximation if the pumping pulse for the single-photon generator is chosen appropriately $[10]$.

Similar to the problem of photon-atom scattering [5], in our work, we focus on the case of the photon scattered perpendicular to the incident direction, i.e., $\theta=\pi / 2$. We have $B(\vec{q}, t \rightarrow \infty) \rightarrow 0$, and $A(\vec{q}, \vec{k}, t \rightarrow \infty)=A_{\pi / 2}$.

$$
A_{\pi / 2}=\frac{N \exp \left[-\left(\Delta q_{x}-\frac{\hbar k_{0}}{m c} \Delta k_{x}\right)^{2} / \eta_{x}{ }^{2}\right]}{\left(\Delta k_{x}+\Delta q_{x}+\frac{\hbar k_{0}{ }^{2}}{2 m \Gamma}+i\right)\left[\left(\Delta k_{x}+\Delta q_{x}\right) / \tau_{z}+i\right]},
$$

where $\Gamma=\pi \sum_{\vec{k}}|g(\vec{k})|^{2} \delta\left(\omega_{e}-c k\right)$ is the exciton's radiative linewidth, $\Delta k_{i} \equiv\left(k_{i}-k_{0}\right) / \Gamma / c$, $\Delta q_{i} \equiv\left(\hbar k_{0} / m \Gamma\right) /\left(q_{i}-k_{0}\right), \eta_{i} \equiv \delta q_{i} \hbar k_{0} / m \Gamma, \tau_{i} \equiv \delta k_{i} / \Gamma / c$ with $i=x, y, z$, are all defined dimensionless parameters. Note that $\eta_{x}$ and $\tau_{z}$ contain all the physical parameters that determine the nature of the exciton-photon system and thus can be treated as physical control parameters for the exciton and the photon, respectively.

We neglect tiny terms in Eq. (7) due to $\hbar k_{0}{ }^{2} \ll m \Gamma$ and $\hbar k_{0} \ll m c$ in realistic conditions.

$$
A_{\pi / 2} \approx \frac{N \exp \left[-\left(\Delta q_{x} / \eta_{x}\right)^{2}\right]}{\left(\Delta k_{x}+\Delta q_{x}+i\right)\left[\left(\Delta k_{x}+\Delta q_{x}\right) / \tau_{z}+i\right]}
$$

with $N$ is the normalization factor

$$
N^{2}=\sqrt{2}\left(1+\tau_{z}\right) / \pi^{3 / 2} \tau_{z} \eta_{x} .
$$

From Eq. (8) and Fig. 2, one sees that the variables $\Delta q_{x}$ and $\Delta k_{x}$ play the symmetric role of the two Lorentzian functions. It makes the probability amplitude $\left|A_{\pi / 2}\right|^{2}$ localized along the diagonal of the momentum space, which implies the non-factorization of the photon-exciton wave function, and then it will generate entanglement between the two particles.

\section{AMPLITUDE ENTANGLEMENT IN MOMENTUM}

In both theoretical and experimental studies $[6,13]$, the ratio (denoted by " $R$ ") of the unconditional and the conditional variances plays a important role, since it is direct 


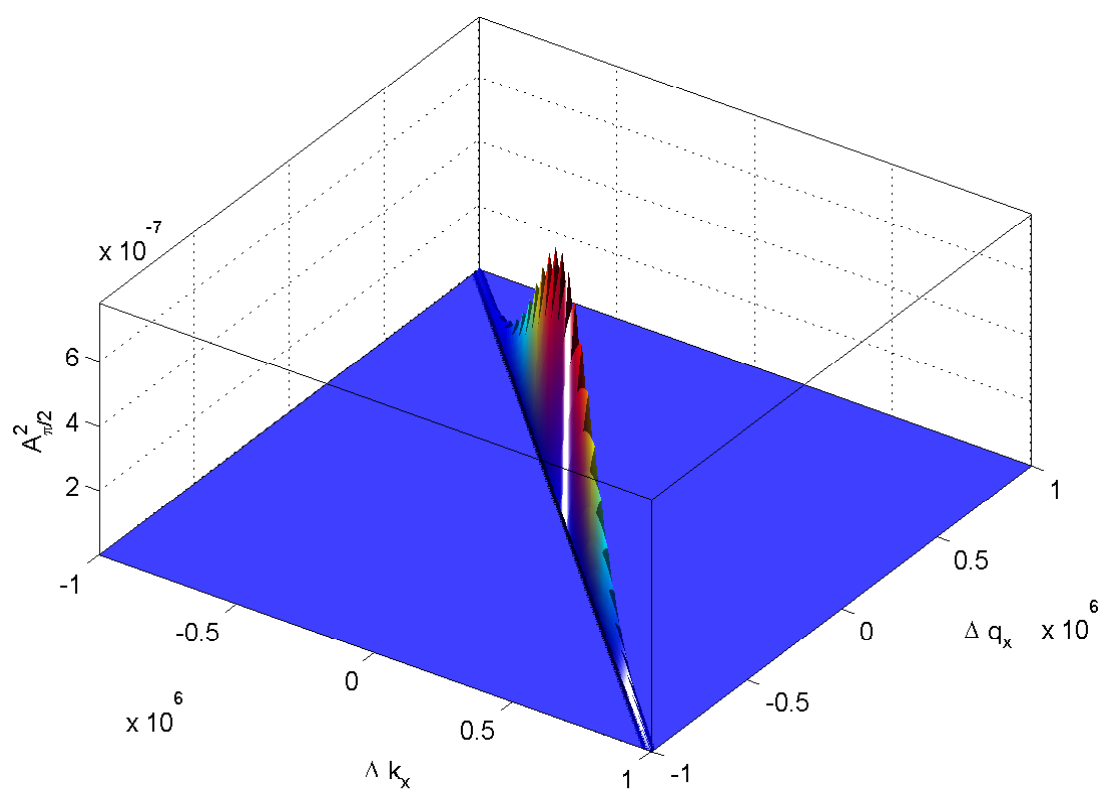

Fig. 2. Plots of amplitude $\left|A_{\pi / 2}\right|^{2}$ with the conditions $\tau_{z}=1, \eta_{x}=10^{6}$

experimental measure of nonseparability (entanglement) of the system. For the singleparticle measurement, the unconditional variance for the effective excitonic momentum is determined as

$\delta^{2} \Delta q_{x}^{\text {single }}=\left\langle\Delta q_{x}{ }^{2}\right\rangle-\left\langle\Delta q_{x}\right\rangle^{2}=\int d \Delta k_{x} d \Delta q_{x} \Delta q_{x}{ }^{2}\left|A_{\pi / 2}\right|^{2}-\left(\int d \Delta k_{x} d \Delta q_{x} \Delta q_{x}\left|A_{\pi / 2}\right|^{2}\right)^{2}$.

Meanwhile, where the photon is previously detected at some known $\Delta k_{x}$, the coincidence measurement gives the conditional variance as

$$
\delta^{2} \Delta q_{x}{ }^{\text {coinc }}=\left\langle\Delta q_{x}{ }^{2}\right\rangle_{\Delta k_{x}}-\left\langle\Delta q_{x}\right\rangle_{\Delta k_{x}}^{2}=\frac{\int d \Delta q_{x} \Delta q_{x}{ }^{2}\left|A_{\pi / 2}\right|^{2}}{\int d \Delta q_{x}\left|A_{\pi / 2}\right|^{2}}-\left(\frac{\int d \Delta q_{x} \Delta q_{x}\left|A_{\pi / 2}\right|^{2}}{\int d \Delta q_{x}\left|A_{\pi / 2}\right|^{2}}\right)^{2}
$$

by using these two variances, we have:

$$
R \equiv \delta \Delta q_{x}{ }^{\text {single }} / \delta \Delta q_{x}{ }^{\text {coinc }} \geq 1 .
$$

Substituting Eqs. (9) and (10) into the definition of $R$, we yield $R\left(\eta_{x}, \tau_{z}\right)$ as a function of parameters $\eta_{x}$ and $\tau_{z}$, the result of which is illustrated in Fig. 3 with $\Delta k_{x}$ fixed at the origin.

In Fig. 3(a), we showed that the entanglement increases monotonously with increasing $\eta_{x}$ or decreasing $\tau_{z}$, which indicates that higher entanglement can be achieved 


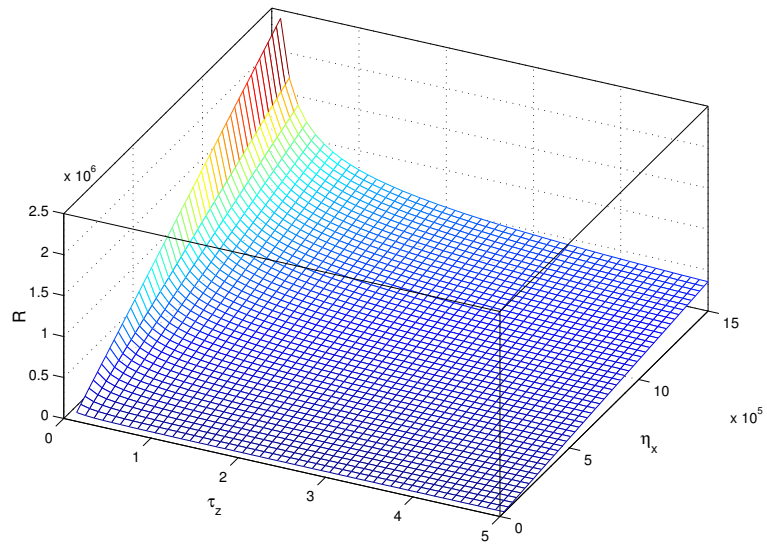

(a)

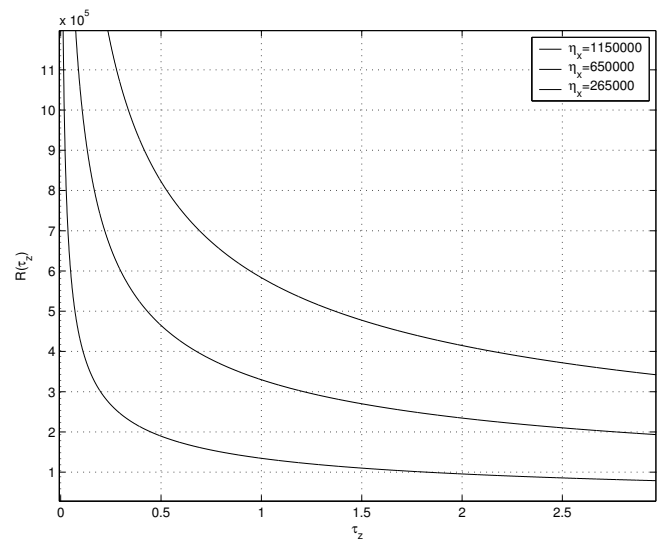

(b)

Fig. 3. (a) Relation between $R$ and the two control parameters $\left(\eta_{x}, \tau_{z}\right)$, (b) Sectional views of (a), with $\eta_{x}=2.65 \times 10^{5}, 6.5 \times 10^{5}, 11.5 \times 10^{5}$ from bottom to top. The ratio $R$ is calculated from variable $\Delta q_{x}$ with $\Delta k_{x}$ is fixed at the orgin

by squeezing the linewidth of the incident photon or broadening the wave packet of the exciton. In particular, due to $\eta_{x} \gg 1$ and $\tau_{z} \ll \eta_{x}$, we have

$$
R \approx \frac{\eta_{x}+\sqrt{\frac{2}{\pi}} \tau_{z}}{2 \sqrt{\tau_{z}}} .
$$

The entanglement increases linearly with increasing $\eta_{x}$, and increases abruptly enhanced with decreasing $\tau_{z}$.

The ratio $R$, which can be experimentally obtained by comparing the momentum dispersion variance, is an appropriate quantification for the entanglement contained in the probability amplitude correlation (thus can be seen as an evaluation of the "amplitude entanglement"). Next, we can see that it reveals a correct varying tendency for the entanglement with its control parameters. However, the definition of $R$ is dependent on its representation space and different choices for the basis of Hilbert space will cause distinct values of $R$. This is because the $R$ ratio is only constructed from the amplitude information of the wavefunction, and then all entanglements included in phase $[7,11]$ are lost. To obtain the "total entanglement", we calculate the Schmidt number $[8,9]$ and compare it with the entanglement ratio $R$ in the next section.

\section{SCHMIDT DECOMPOSITION AND FULL ENTANGLEMENT}

In order to evaluate the full entanglement for the bi-partite system in a pure state, we use the "Schmidt number". Mathematically, the entanglement of an unfactorable wave function can be completely characterized by the Schmidt number, which is denoted by the 
value $K \equiv\left(\sum_{n=0}{ }^{\infty} \lambda_{n}{ }^{2}\right)^{-1}$, where $\lambda_{n}$ are the eigenvalues of the integral equation:

$$
\int d \Delta q_{x}^{\prime} \rho^{e}\left(\Delta q_{x}, \Delta q_{x}^{\prime}\right) \psi_{i}\left(\Delta q_{x}^{\prime}\right)=\lambda_{i} \psi_{i}\left(\Delta q_{x}\right)
$$

On the other hand, the Schmidt number is expressed via trace of squared partial density matrix, $K=1 / \operatorname{Tr}\left(\left(\rho^{e}\right)^{2}\right)=1 / \operatorname{Tr}\left(\left(\rho^{p}\right)^{2}\right)\left(\rho^{e}, \rho^{p}\right.$ are the partial density matrix of the exciton and the photon, respectively).

The partial density matrix for the exciton is defined by

$$
\rho^{e}\left(\Delta q_{x}, \Delta q_{x}^{\prime}\right)=\int d \Delta k_{x} A_{\pi / 2}\left(\Delta q_{x}, \Delta k_{x}\right) A_{\pi / 2}^{*}\left(\Delta q_{x}^{\prime}, \Delta k_{x}\right)
$$

Substituting Eq. (8) into Eq. (13), we have:

$$
\rho^{e}\left(\Delta q_{x}, \Delta q_{x}^{\prime}\right)=-\frac{4 \pi i N^{2} \tau_{z}^{2} e^{-\frac{\left(\Delta q_{x}^{2}+\Delta q_{x}^{\prime 2}\right)}{\eta x^{2}}}}{\left.\left.\left(\Delta q_{x}-\Delta q^{\prime}{ }_{x}+2 i\right)\left(\Delta q_{x}-\Delta q_{x}^{\prime}\right)+2 \tau_{z} i\right)\left(\Delta q_{x}-\Delta q^{\prime}{ }_{x}+\left(\tau_{z}+1\right) i\right)\right)} .
$$

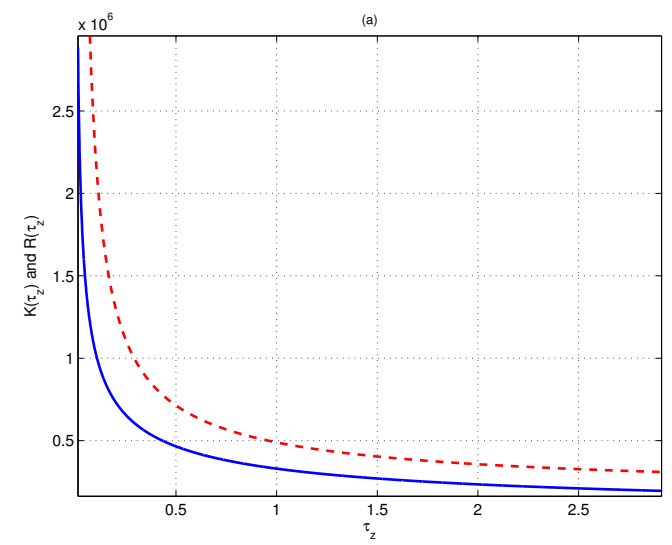

(a)

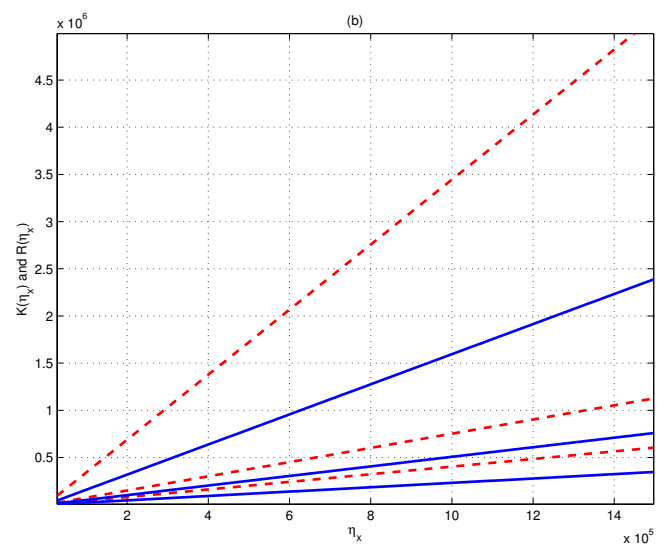

(b)

Fig. 4. (a) Schmidt number $K$ and amplitude entanglement degree $R$ in dependence on $\tau_{z}$ with $\eta_{x}=6.5 \times 10^{5}$, (b) Relation between $K$ and $R$ in the dependence on $\eta_{x}$ with $\tau_{z}=.1,1,5$, spots line is for $K$ whereas solid line is plotted for $R$

Finally, we obtained an analytical formula for the Schmidt number, which has a simple asymptotic form:

$$
K \approx \frac{\eta_{x}\left(\tau_{z}+3\right)\left(3 \tau_{z}+1\right)}{6 \sqrt{\pi}\left(\tau_{z}+1\right) \tau_{z}}
$$

From Fig. 4 we find that, similar to the ratio $R, K$ rises linearly with parameter $\eta_{x}$ and will increase rapidly when the linewidth of the incident photon is squeezed narrower to the exciton linewidth. Second, when $\tau_{z}$ is fixed, the slope of $K\left(\eta_{x}\right)$ is always much larger than that of $R\left(\eta_{x}\right)$, which means that more entanglement information will transfer to the 
phase when $\eta_{x}$ becomes larger [5,7], and this phenomenon will become more evident when $\tau_{z}$ reduced.

\section{CONCLUSION}

In summary, we have investigated the recoiled induced excitonphoton entanglement in the scattering. To evaluate the entanglement, first we use an experimentally accessible parameter $R$, which denotes the ratio between momentum variance in single particle and in coincidence observations, second we use standard Schmidt decomposition to reveal the full entanglement information. Because specific parameters of excitons are different from those of atoms, the physical control parameters in the new scheme are calculated in much higher scales. Compared with the degree of entanglement between atom and photon [3-6] the new scheme produces super-high momentum entanglement between a single exciton and a single photon. Moreover, we obtained for the first time a simple analytical formula of $\mathrm{K}$, which could be used to demonstrate explicitly the dependence of entanglement on the control parameters. However, there are still difficulties in the new scheme such as the

weak stability of excitons in semiconductors and decoherence due to effects of environment, which can cause the disentanglement [7].

\section{REFERENCES}

[1] A. Einstein, B. Podolsky, N. Rosen, Phys. Rev. 47 (1935) 777; J. C. Howell et al., Phys. Rev. Lett. 92 (2004) 210403.

[2] S. L. Braunstein and P. V. Loock, Rev. Mod. Phys. 77 (2005) 513.

[3] K. W. Chan, C. K. Law, and J. H. Eberly, Phys. Rev. Lett. 88 (2002) 100402.

[4] K. W. Chan et al., Phys. Rev. A68 (2003) 022110.

[5] R. Guo and H. Guo, Phys. Rev. A 73 (2006) 012103.

[6] M. D. Reid and P. D. Drummond, Phys. Rev. Lett. 60 (1988) 2731; Michael S. Chapman et al., Phys. Rev. Lett. 75 (1995) 3783; Christian Kurtsiefer et al., Phys. Rev. A 55 (1997) R2539.

[7] R. Guo and H. Guo,e-print quant-ph/0701018v2, e-print quant-ph/0611205v2(2007)

[8] M.Karelin, e-print quant-ph/0606055v2(2006)

[9] S. Parker, S. Bose, M.B. Plenio,e-print quant-ph/9906098v2(2006)

[10] Matthias Keller et al., Nature (London) 431 (2004) 1075; M.Keller et al., New J. Phys. 6 (2004) 95; J. McKeever et al., Science 303 (2004) 1992; S. Brattke, B. T. H. Varcoe, and H.Walther, Phys. Rev. Lett. 86 (2001) 3534.

[11] K. W. Chan and J. H. Eberly, e-print quant-ph/0404093 (2004).

[12] A. Ekert and P. L. Knight, Am. J. Phys. 63 (1995) 415; S. Parker, S. Bose, and M. B. Plenio, Phys. Rev. A61 (2000) 032305.

[13] M. V. Fedorov et al., Phys. Rev. A 69 (2004) 052117.

Received 15 August 2008. 